\title{
1 Determination of salivary cortisol in donkey stallions
}

\section{Short title: Salivary cortisol in donkeys}

3 Francesca Bonelli ${ }^{\mathrm{a}}$, Alessandra Rota ${ }^{\mathrm{a}}$, Christine Aurich ${ }^{\mathrm{b}}$, Natascha Ille ${ }^{\mathrm{b}}$, Francesco Camillo ${ }^{\mathrm{a}}$, Duccio

$4 \quad$ Panzani $^{\mathrm{a}}$, Micaela Sgorbini ${ }^{\mathrm{a}}$

5 aDepartment of Veterinary Sciences, via Livornese snc, 56122 San Piero a Grado (PI), Italy

$6{ }^{b}$ Centre for Artificial Insemination and Embryo Transfer, University of Veterinary Sciences, Vienna,

7 Austria.

9 Corresponding Author:

10 Micaela Sgorbini

11 Department of Veterinary Sciences

12 via Livornese snc

1356122 San Piero a Grado (PI), Italy

14 francesca.bonelli@unipi.it

\section{Abstract}

Salivary cortisol provides information about free plasma cortisol concentration and

18 salivary sampling is a non-invasive well-tolerate procedure. The aim of this study was to validate

19 a commercial enzyme immunoassay for the determination of salivary cortisol in donkeys.

20 Saliva samples were collected in 4 donkey stallions on thirteen non-consecutive days at

21 8:30 AM to avoid circadian variation. Animals were already accustomed to be handled. Saliva

22 was collected by using a swab inserted at the angle of the lips, placed onto the tongue for 1 min

23 and returned into a polypropylene tube. Tubes were centrifuged and at least $1 \mathrm{ml}$ of saliva was 
24 aspirated from each sample and frozen at $-20^{\circ} \mathrm{C}$ until analysis. A commercial enzyme

25 immunoassay kit without extraction was used for determination of cortisol in saliva. Median

26 cortisol concentrations with minimum and maximum value were calculated.

27 Recovery of cortisol standard in donkey saliva was $107.9 \%$ and serial dilution of donkey

28 saliva samples with assay buffer resulted in changes in optical density parallel to the standard

29 curve. Cross-reactivity of the antiserum was $10.4 \%$ with 11 -deoxycortisol, $5.2 \%$ with

30 corticosterone, $0.4 \%$ with 11 -deoxycorticosterone, $0.2 \%$ with cortisone and $<0.1 \%$ with

31 testosterone, progesterone and estradiol. The intra-assay coefficient of variation was $10.7 \%$, the

32 inter-assay variation was $8.0 \%$ and the minimal detectable concentration was $0.01 \mathrm{ng} / \mathrm{ml}$.

33 The results of the present study demonstrate the validity of a commercial kit to determine

34 the concentration of cortisol in donkey saliva, as already reported in other species.

35 Key words: donkey, salivary cortisol, validation, stress, enzyme immunoassay. 


\section{Introduction}

Domestic animals react to stress through physiological responses, which are the results of

38 individual emotional reactivity. [1] The hypothalamic-pituitary-adrenocortical (HPA) system is

39 activated in response to stressors. While early response is under catecholaminergic control, late

40 physiological response to stressor is commonly assessed by determination of glucocorticoid

41 concentrations, such as cortisol.

42 An increase in hypothalamic-pituitary-adrenocortical activity indicates a physiological response

43 to different stressors, and measurement of plasma corticosteroids is frequently used to study

44 middle and longtime stress response. [2]

45 Cortisol was primarily obtained from blood, plasma or urine. Blood sampling itself (by syringe or

46 by catheterization) can produce stress in the animal causing a rise in cortisol levels. [3,4] Urine

47 and fecal samples can be easily and non-invasively collected without submitting animals to

48 stress, [5,6] but the volume/unit weight varies between animals and species [5] and time periods

49 between application of the stressor and collection of the urine or feces cannot be easily

50 controlled. On the other hand the animals are hardly affected by saliva sample collection, $[7,8]$

51 thus saliva can be considered a non-invasive monitoring of HPA functioning in animals.

52 Cortisol is passively diffused into the salivary glands, not bound to proteins and its concentration

53 is not affected by salivary flow rate. Therefore, the salivary cortisol provides direct information

54 about free plasma cortisol concentration, which is the only biologically active fraction in the

55 organism, owing to it being able to bind to cell receptors. $[9,10]$

56 Furthermore, the collection of saliva is a non-invasive procedure, which animals usually tolerate

57 better than blood sampling. For all of these reasons, the interest in measurement of salivary

58 cortisol concentration in animals has increased during the past few years. 
59 Salivary cortisol concentration has been evaluated in human, [8,11-12] dogs, [13-16] cattle,

60 [3,17-19] pigs [20-21] and horses. [9-10,22-25]

61 In the last decades other equids, the donkey, achieve a relevant position in the human society

62 thanks to their employment in animal-assisted therapy [26] and milk production. [27-29]

63 Therefore, the welfare and stress responses of donkey become an interesting field of research

64 related to its behavior. To the authors' knowledge there are no validated commercial kits for the

65 determination of salivary cortisol levels in donkeys. We followed the hypothesis that

66 determination of cortisol concentration in donkey saliva is possible. It was the aim of this study

67 to validate a commercial enzyme immunoassay for the determination of salivary cortisol 68 concentrations in donkeys.

70 Materials and methods

71 This study was conducted between September 2015 and February 2016 and was approved by the

72 Ethical Committee (OPBA) of the University of Pisa, according to D.lvo 26/2014. Four Amiata

73 donkey stallions (jacks), aged 3 years (born between May and July 2012) were included in this

74 study.

75 The jacks were kept together in a paddock $(10 \times 20 \mathrm{mt})$ for three months (September-November

76 2015). Then donkeys were housed for three months (December 2015-January 2016) in single

77 boxes $(3.5 \times 3.5 \mathrm{mt})$ with a small outside paddock $(3.5 \times 6 \mathrm{mt})$, where they could see each other,

78 but had no physical contact. Boxes were daily cleaned and bedded with straw. All the donkeys

79 had free access to water from a water bowl and were fed meadow hay ad libitum and commercial

80 concentrate $\left(\right.$ Equifioc $^{\circledR}$, Molitoria Val di Serchio, Lucca, Italy), according NRC recommendations

81 for adult donkeys. [30] Weight was $280.3 \pm 30.4 \mathrm{~kg}$ when donkeys were moved to boxes and 
$82 \quad 283.5 \pm 27.0 \mathrm{~kg}$ at the end of the study, with a difference of $-3,+1,+10$ and $+5 \mathrm{~kg}$ in the single

83 animals, while Body Condition Score (BCS) was always evaluated between 5 and 6/9. [31]

84 Saliva samples were collected on thirteen non-consecutive days at 8:30 AM to avoid circadian

85 variation [10] using a cotton-based swab (Salivette ${ }^{\circledR}$, Sarstedt, Numbrecht-Rommelsdorf,

86 Germany) as described in horses. [22-23] Sampling time for cortisol evaluation was scheduled in

87 line with another project running simultaneously. [32] Briefly, all the 4 jacks underwent to semen

88 collections thrice weekly (Tuesday, Thursday and Saturday) during 3 weeks in the month of

89 November 2015 and February 2016. One saliva sampling took place the week before the

90 beginning of semen collection (November 2015), while the other 12 samplings were carried out

91 every Tuesday and Saturday when semen collection was scheduled (6 samplings in November

922015 and 6 samplings in February 2016). Thus, 7 saliva sampling were done in November 2015

93 and 6 in February 2017.

94 Samplings were always made before feeding and cleaning procedures of the boxes and before

95 semen collection, when scheduled, with animals were at rest. Special care was used to minimize

96 stress during the sampling procedure, in particular, the same operator handled the animals

97 throughout the study and any special restraint during saliva samplings was applied. The swab was

98 grasped with a surgical clamp, inserted at the angle of the lips into the mouth of the donkey and

99 placed gently onto the tongue for $1 \mathrm{~min}$ and afterwards returned into a polypropylene tube. Tubes

100 were then centrifuged for $10 \mathrm{~min}$ at $700 \mathrm{~g}(1800 \mathrm{~g})$, at least $1 \mathrm{ml}$ of saliva was aspirated from each

101 sample and the obtained saliva was frozen at $-20^{\circ} \mathrm{C}$ until analysis.

102 A commercial enzyme immunoassay kit without extraction (Demeditec Diagnostics, Kiel-

103 Wellsee, Germany) was used for determination of cortisol in saliva. The assay was validated for

104 asinine saliva in the laboratory of the unit for Obstetrics, Gynecology and Andrology,

105 Department for Small Animals and Horses, Vetmeduni (Vienna, Austria). Cross-reactivity of the 
106 antiserum was determined at 50\% binding with 11-deoxycortisol, corticosterone, 11-

107 deoxycorticosterone, cortisone, testosterone, progesterone and estradiol-17 $\beta$. Recovery of cortisol

108 standards added to donkey saliva was tested. Parallelism between serial dilutions of saliva

109 samples with assay buffer and respective changes in optical density with respect to the standard

110 curve were determined. The intra-assay and inter-assay coefficients of variation were determined

111 from duplicates of control saliva with low and high cortisol concentrations run in each assay

$112(\mathrm{n}=5)$. The minimal detectable concentration was defined as 2 standard deviations from zero

113 binding.

114 The Graph Pad Prism 6 (USA) software was used for statistical analysis. Data were analyzed for

115 normal distribution by Kolmogorov-Smirnov test. Because not all data were normally distributed,

116 results were expressed as median values of cortisol concentration and range calculated for each

117 day of sampling. Values are given as $\mathrm{ng} / \mathrm{ml}$.

\section{Results}

120 Saliva samples could be easily collected in all the donkeys enrolled in this study. The sampling

121 procedure was well accepted by all the animals. In one donkey (n. 4 table 1), the amount of saliva

122 collected was occasionally too small for analysis (samples 2nd, 6th and 9th).

123 Recovery of cortisol standard in donkey saliva was $107.9 \%$ and serial dilution of donkey saliva

124 samples with assay buffer resulted in changes in optical density parallel to the standard curve.

125 Cross-reactivity of the antiserum at 50\% binding was $10.4 \%$ with 11 -deoxycortisol, $5.2 \%$ with

126 corticosterone, $0.4 \%$ with 11 -deoxycorticosterone, $0.2 \%$ with cortisone and $<0.1 \%$ with

127 testosterone, progesterone and estradiol. The intra-assay coefficient of variation was $10.7 \%$, the

128 inter-assay variation was $8.0 \%$ and the minimal detectable concentration was $0.01 \mathrm{ng} / \mathrm{ml}$. 
129 Results of cortisol concentrations determined in saliva collected from donkeys on different days

130 are presented in Table 1.

131

\begin{tabular}{|c|c|c|c|c|c|c|c|c|c|c|c|c|c|}
\hline & \multicolumn{10}{|c|}{ Salivary cortisol concentration (ng/ml) } \\
\hline Donkeys & I & II & III & IV & V & VI & VII & VIII & IX & X & XI & XII & XIII \\
\hline 1 & 3.9 & 3.6 & 10.8 & 3.6 & 6.2 & 1.5 & 4.6 & 3.4 & 5.8 & 5.4 & 4.6 & 5.6 & 4.8 \\
\hline 2 & 0.5 & 7.25 & 8.7 & 8.11 & 7.5 & 1.73 & 1.7 & 4.7 & 5.9 & 2.9 & 8.1 & 1.9 & 1.9 \\
\hline 3 & 9.4 & 5.36 & 20.6 & 7.72 & 1.0 & 6.6 & 2.8 & 3.7 & 9.2 & 3.0 & 5.0 & 6.9 & 2.1 \\
\hline 4 & 3.1 & $*$ & 4.7 & 10.38 & 9.5 & $*$ & 9.7 & 5.6 & $*$ & 3.7 & 11.8 & 4.2 & 2.7 \\
\hline Median & 3 & 5 & 9 & 7.5 & 6.6 & 1 & 3 & 3.5 & 5 & 3 & 6.5 & 4.5 & 2 \\
\hline Range & $0-9$ & $3-7$ & $4-20$ & $3-10$ & $1-9$ & $1-6$ & $1-9$ & $3-5$ & $5-9$ & $2-5$ & $4-11$ & $1-6$ & $1-4$ \\
\hline
\end{tabular}

132

133 Tab 1. Results of salivary cortisol concentration obtained in 4 individual donkey stallions on

13413 non-consecutive days. Legend: *Amount of saliva not sufficient for cortisol analysis.

136 Discussion

137 The production of cortisol is regulated by the corticotrophic axis and can be altered in different

138 circumstances. Cortisol concentrations can rise in stressful situations, thus it is considered to be

139 an indicator of animal welfare [4] Although traditionally cortisol concentrations have been

140 determined in plasma or serum, their determination in samples easily collectable under field

141 conditions (saliva, milk, urine, feces and hair) can be of interest for animal welfare studies in

142 different species. [8,33-37] Thus, in our study, a commercial kit assay system, originally used in

143 humans for measuring concentration of cortisol, was adapted and validated for donkey saliva

144 samples.

145 The evaluation of salivary cortisol has some advantages in large animals. Cortisol concentration

146 in saliva corresponds to the free fraction of cortisol in plasma, thus salivary cortisol represents a

147 better indicator of the possible effects of the corticotrophic axis on the animal organism than 
148 plasma cortisol. [17] Moreover, blood collection always produces stress in the animal that can

149 cause cortisol levels to rise. [7-8] On the other hand, saliva sampling, especially in already

150 handled animals, can be an easy and stress-free procedure as confirmed by our results. All

151 donkeys included in the study well tolerated the procedures and the amount of saliva collected

152 was good enough in the majority of the samples. The results of the present study demonstrate the

153 validity of a commercial kit to determine the concentration of cortisol in donkey saliva, as

154 already reported in dogs [13-15] and horses. [9-10,22-23,38]

155 In the specificity tests, all the cross reactions observed were in line with the manufactory

156 performance characteristic declared. A very low cross-reaction has been found with 11-

157 deoxycortisol (10.4\%) and corticosterone (5.2\%). The 11-deoxycortisol is a direct precursor of

158 cortisol and is found in plasma at lower concentrations than cortisol. Literature showed that the

159 11-deoxycortisol have none glucocorticoid effect and almost no mineral corticoid effect. [17]

160 Corticosterone is the predominant glucocorticoid produced in some species (i.e. mice, rats and

161 birds). [39] Even if corticosterone is also synthesized in cortisol-dominant species, like equids, its

162 glucocorticoid activity in these species is still under assessment. [39] Cross-reactivity with 11-

163 deoxycorticosterone, cortisone and with testosterone, progesterone and estradiol were negligible.

164 Intra- and inter-assay coefficients of variation are used to control the potential analytic error in

165 biological assay systems. [40] Our results showed a slightly increased intra- and inter-assay CV

$166 \%$ compared with the manufactory performance characteristic declaration (intra-assay CV of 3.8-

$1675.8 \%$ and inter-assay CV of 6.4-6.2\%). However, the validation results indicated that the method

168 was adequate for salivary cortisol measurements given that it is generally accepted that the CV

169 must be lower than $20 \%$ for analytical determinations. [40] The minimal detectable concentration

170 found in our study was slightly below those indicated by the manufactory guidelines $(0.01 \mathrm{ng} / \mathrm{ml}$

171 vs $0.024 \mathrm{ng} / \mathrm{ml}$, respectively) and published in cows, [17,41] but similar to results in pigs [20] and 
172 horses. [10]

173

\section{Conclusions}

175 In conclusion, given the advantages of the stress-free collection and processing of saliva and the

176 good performance of the assay, the use of cortisol detection in saliva could be considered highly

177 suitable in donkey stress research. A limit of the present study is the fact that we did not compare 178 salivary cortisol concentrations to concentration of cortisol in blood collected at the same time.

179 However, when this was done in horses, the relationship was very good. [9-10] Also, despite in

180 the majority of the samples, the quantity of saliva was good enough for running the assay, an

181 improvement in saliva collection procedure need to be addressed.

182

183 Acknowledgements

184 We are grateful to Ente Terre Regionali Toscane for allowing us to employ the animals for this 185 study. 


\section{References}

187 1. Koolhaas JM, De Boer SF, Coppens CM, Buwalda B. Neuroendocrinology of coping 188 styles: towards understanding the biology of individual variation. Front. Neuroendocrinol. $189 \quad 2010 ; 31: 307-321$.

2. Pacak K, Palkovits M. Stressor specificity of central neuroendocrine responses: Implications for stress-related disorders. Endocr Rev. 2001;22:502-548.

3. Fell LR, Shutt DA. Adrenocortical response of calves to transport stress as measured by salivary cortisol. Can J Anim Sci. 1986;66:637-641.

4. Cook NJ, Schaefer AL, Lepage P, Morgan Jones S. Salivary vs. serum cortisol for the assessment of adrenal activity in swine. Can J Anim Sci. 1996;76:329-335. via faeces and urine in domestic livestock. Anim Reprod Sci. 1996;43:43-63.

6. Mostl E, Palme R. Hormones as indicators of stress. Dom Anim Endocrinol. 2002;23:6774.

7. Fell LR, Shutt DA, Bentley CJ. Development of a salivary cortisol method for detecting changes in plasma "free" cortisol arising from acute stress in sheep. Aust Vet J. $1985 ; 12: 403-406$. assay for cortisol in the saliva of man and domestic farm animals. J Endocrinol. 1989;123: R13-R16. serum and salivary cortisol concentrations in horses using an adrenocoticotropic hormone challenge. Equine Vet J. 2011;43:487-493. 
10. Aurich J, Wulf M, Ille N, Erber R, von Lewinski M, Palme R, et al. Effects of season, age, sex, and housing on salivary cortisol concentrations in horses. Domest Anim Endocrinol. 2015;52:11-16.

11. Aardal E, Holm AC. Cortisol in Saliva. Reference ranges and relation to cortisol in serum. Eur J Clin Chem Clin Biochem. 1995;33:927-932.

12. Turpeinen U, Hamalainen E. Determination of cortisol in serum, saliva and urine. Best Pract Res Clin Endocrinol Metab. 2013;27(6): 795-801. hour period in dogs. Horm Metab Res. 2003;35:355-357. Ruckstuhl NS. Salivary cortisol concentrations in healthy dogs and dogs with hypercortisolism. J Vet Intern Med. 2010;24:551-556. pigment differences. Domest Anim Endocrinol. 2010;39(3):171-180. dogs. J Chromatogr B Analyt Technol Biomed Life Sci. 2016;1028:192-198. Validation of an EIA technique for the determination of salivary cortisol in cattle. Span J Agric Res. 2004;2(1):45-51. Cattle After ACTH Administration and Milking. J Dairy Sci. 2004;87:1713-1718. 
stressful procedure in dairy cattle. Acta Vet Scand. 2014;56:61. doi: 10.1186/s13028-0140061-3.

20. Escribano D, Fuentes-Rubio M, Cerón JJ. Validation of an automated chemiluminescent immunoassay for salivary cortisol measurements in pigs. J Vet Diagn Invest. 2012;24(5): 918-923.

21. Escribano D, Gutiérrez AM, Tecles F, Cerón JJ. Changes in saliva biomarkers of stress and immunity in domestic pigs exposed to a psychosocial stressor. Res Vet Sci 2015;102: 38-44. Changes in cortisol release and heart rate variability in sport horses during long-distance road transport. Domest Anim Endocrinol. 2010a;38:179-189. rate variability in horses during road transport. Horm Behav. 2010b;57:209-215. the circadian rhythm of the serum and salivary cortisol concentrations in the horse. Domest Anim Endocrinol. 2013;45:38-42. exercised throughbred horses. J Equine Vet Sci. 2013;33(12):1106-1109. equestrian therapy and onotherapy in physical and psycho-social performances of adults with intellectual disability: a preliminary study of evaluation tools based on the ICF classification. Disabil Rehabil. 2012;34(4): 279-297.

27. Carroccio A, Cavataio F, Montalto G. Intolerance to hydrolysed cow's milk proteins in infants: clinical characteristic and dietary treatment. Clin Exp Allergy. 2000;30:1597- 
1603.

28. Muraro MA, Giampietro PG, Galli E.. Soy formulas and non bovine milk. Ann Allergy Asthma Immunol. 2002;89(S):97-101.

29. Camillo F, Rota A, Biagini L, Tesi M, Fanelli D, Panzani D. The Current Situation and Trend of Donkey Industry in Europe. J Equine Vet Sci. 2018;65:44-49.

30. National Research Council. Nutrient requirements of horses: sixth revised edition. Washington, DC: The National Academies Press; 2007.

31. Henneke DR, Potter GD, Kreider JL, Yeates BF. Relationship between condition score, physical measurements and body fat percentage in mares. Equine Vet J. 1983;15(4): 371372.

32. Rota A, Sgorbini M, Panzani D, Bonelli F, Baragli P, Ille, N et al. Effect of housing milk cortisol concentration as a measure of short term stress response in lactating dairy cows. Anim Welfare. 1998;7:77-86.

34. Cirimele V, Kintz P, Dumestre V, Goullé JP, Ludes B. Identification of ten corticosteroids in human hair by liquid chromatography-ionspray mass spectrometry. Forensic Sci Internat. 1999;107(1-3):381-388. determination of natural profiles of androgens, progestogens and glucocorticoids in 
37. Palme R, Robia C, Baumgartner W, Möstl E. Transport stress in cattle as reflected by a increase in faecal cortisol metabolite concentration. Vet. Rec. 2000;146:108-109. cortisol concentrations in foals at birth. Vet J. 2015;203:250-252.

39. Gong S, Miao Y-L, Jiao G-Z, Sun M-J, Li H, Lin J, et al. Dynamics and Correlation of Serum Cortisol and Corticosterone under Different Physiological or Stressful Conditions in Mice. PLoS ONE. 2015;10(2):e0117503.

40. Food and Drug Administration (FDA), Center for Drug Evaluation and Research (CDER), and Center for Veterinary Medicine (CVM). Guidance for industry: Bioanalytical method validation. Washington, DC: U.S. Department of Health and Human Services. 2001; Retrieved from http://www.fda.gov/downloads/Drugs/Guidance ComplianceRegulatoryInformation/Guidances/ucm070107.pdf Helmond FA. Responses of young calves on two levels of feeding to transportation. J Anim Sci. 1996;63:79-89. 


\begin{tabular}{|c|c|c|c|c|c|c|c|c|c|c|c|c|c|}
\hline & \multicolumn{10}{|c|}{ Salivary cortisol concentration (ng/ml) } \\
\hline Donkeys & I & II & III & IV & V & VI & VII & VIII & IX & X & XI & XII & XIII \\
\hline 1 & 3.9 & 3.6 & 10.8 & 3.6 & 6.2 & 1.5 & 4.6 & 3.4 & 5.8 & 5.4 & 4.6 & 5.6 & 4.8 \\
\hline 2 & 0.5 & 7.25 & 8.7 & 8.11 & 7.5 & 1.73 & 1.7 & 4.7 & 5.9 & 2.9 & 8.1 & 1.9 & 1.9 \\
\hline 3 & 9.4 & 5.36 & 20.6 & 7.72 & 1.0 & 6.6 & 2.8 & 3.7 & 9.2 & 3.0 & 5.0 & 6.9 & 2.1 \\
\hline 4 & 3.1 & $*$ & 4.7 & 10.38 & 9.5 & $*$ & 9.7 & 5.6 & $*$ & 3.7 & 11.8 & 4.2 & 2.7 \\
\hline Median & 3 & 5 & 9 & 7.5 & 6.6 & 1 & 3 & 3.5 & 5 & 3 & 6.5 & 4.5 & 2 \\
\hline Range & $0-9$ & $3-7$ & $4-20$ & $3-10$ & $1-9$ & $1-6$ & $1-9$ & $3-5$ & $5-9$ & $2-5$ & $4-11$ & $1-6$ & $1-4$ \\
\hline
\end{tabular}

bioRxiv preprint doi: https://doi.org/10.1101/493965; this version posted December 11, 2018. The copyright holder for this preprint (which was

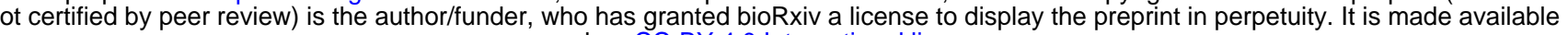

